\title{
Sub-micron optical waveguides for silicon photonics formed via the Local Oxidation of Silicon (LOCOS)
}

\author{
F Y Gardes ${ }^{1}$, G T Reed ${ }^{1}$, A P Knights ${ }^{2}$, G Mashanovich ${ }^{1}$, P E Jessop ${ }^{2}$, \\ L Rowe $^{3}$, S McFaul ${ }^{2}$, D Bruce ${ }^{2}$, N G Tarr ${ }^{3}$ \\ ${ }^{1}$ Advanced Technology Institute, University of Surrey, \\ Guildford, Surrey, GU2 7XH, United Kingdom \\ ${ }^{2}$ Department of Engineering Physics, McMaster University, 1280 Main Street West, \\ Hamilton, Ontario, L8S 4L7, Canada. \\ ${ }^{3}$ Department of Electronics, Carleton University, 1125 Colonel By Drive, \\ Ottawa, Ontario, K1S 5B6, Canada.
}

\begin{abstract}
In this paper we report a novel fabrication technique for silicon photonic waveguides with sub-micron dimensions. The technique is based upon the Local Oxidation of Silicon (LOCOS) process widely utilised in the fabrication of microelectronics components. This approach enables waveguides to be fabricated with oxide sidewalls with minimal roughness at the silicon $/ \mathrm{SiO}_{2}$ interface. It is also sufficiently flexible to enable the depth of the oxidised sidewall to be varied to control the polarisation performance of the waveguides.

We will present preliminary results on submicron waveguide fabrication and loss characteristics (less than $1 \mathrm{~dB} / \mathrm{cm}$ ), as well as effects of varying waveguide width on modal properties of the waveguides. We consider the ease of fabrication, as well as the quality of the devices produced in preliminary experimental fabrication results, and compare the approach to the more conventional requirements of high resolution photolithographically produced waveguides. We also discuss preliminary optical results, as measured by conventional means. Issues such as the origins of loss are discussed in general terms, as are the fabrication characteristics such as waveguide wall roughness and waveguide profile. We will discuss further work that will help to establish the potential of the technique for future applications.
\end{abstract}

Keywords: Silicon-On-Insulator (SOI), silicon photonics, rib waveguides, single-mode condition, LOCOS

\section{INTRODUCTION}

Silicon Photonics based primarily on Silicon-On-Insulator (SOI) is becoming well established for a range of applications [e.g. 1-3]. In this technology the large refractive index difference between core and cladding facilitates strong optical confinement, which in turn allows for relatively small device dimensions so that a higher packing density can be achieved. A further benefit to using this material system comes from the mature field of silicon processing associated with microelectronics. If the technology becomes all pervasive in mass production applications such as optical interconnect and fibre to the home, then a host of other application areas will benefit. However, in this paper we discuss an alternative, very low cost approach to fabricating optical devices and circuits, which would be inherently suitable for relatively small volume applications, and still very low cost.

We have utilised the well-established VLSI technique of the LOCal Oxidation of Silicon (LOCOS) to form rib waveguides in SOI. Micrometer size waveguides using this process have previously been demonstrated by Rowe et al [1] reporting relatively low loss (less than $1 \mathrm{~dB} / \mathrm{cm}$ ), as well as the fabrication of an unbalanced Mach-Zehnder device with Y-splitter junctions capable of a 6-10 dB modulation depth, measured in the 1470-1580 nm wavelength range. In

Silicon Photonics III, edited by Joel A. Kubby, Graham T. Reed

Proc. of SPIE Vol. 6898, 68980R, (2008) · 0277-786X/08/\$18 · doi: 10.1117/12.765383

Proc. of SPIE Vol. 6898 68980R-1 
this paper, we present much smaller LOCOS waveguides in the submicrometer range, as well as discussing the fabrication technique and the measurement of optical loss at $1550 \mathrm{~nm}$ for both TE and TM polarisations.

\section{WAVEGUIDE FABRICATION}

The starting material was a p-type wafer with a $1500 \mathrm{~nm}$ thick silicon overlayer, and a $2.8 \mu \mathrm{m}$ buried oxide layer. The wafer was thinned using thermal oxidation and subsequently etched in buffered HF to reduce the overlayer thickness to around $650 \mathrm{~nm}$. A $40 \mathrm{~nm} \mathrm{SiO}$ layer and a $\mathrm{Si}_{3} \mathrm{~N}_{4}$ masking layer of $80 \mathrm{~nm}$ was deposited by Low Pressure Chemical Vapour Deposition (LPCVD). Trenches in the $\mathrm{Si}_{3} \mathrm{~N}_{4}$ layer were then defined by photolithography and plasma etched. The structure was subsequently wet-oxidized to produce a $410 \mathrm{~nm}$ thick $\mathrm{SiO}_{2}$ layer in the unmasked trench areas. Finally the $\mathrm{Si}_{3} \mathrm{~N}_{4}$ layer was removed to leave an optical waveguide between the oxidized trenches. This process is summarised in figure1, whilst figure 2 shows a Scanning Electron Microscope (SEM) cross section of one of the fabricated waveguides. Significantly, the sidewalls of the waveguide have an angle of $22^{\circ}$ in relation to the substrate, however we note that this angle could be increased significantly by the use of modified LOCOS processes such as SWAMI [2].

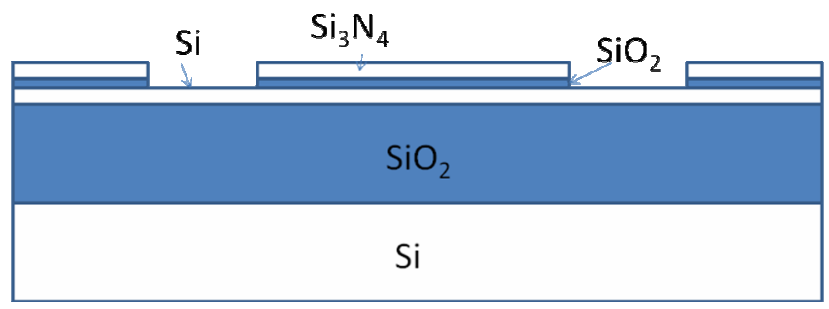

a)

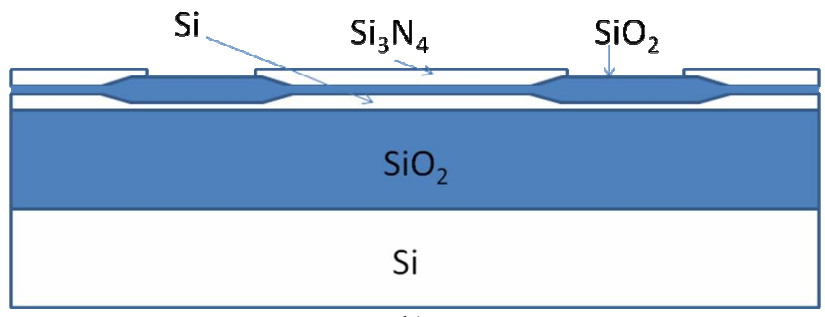

b)

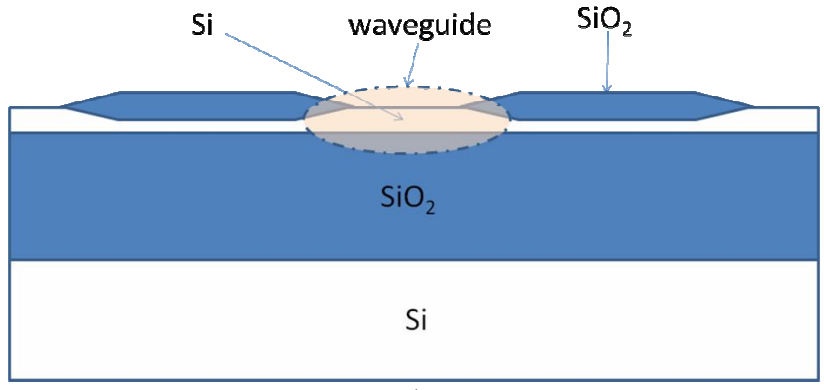

c)

Fig: 1 LOCOS waveguides fabrication process: a) mask formation,b) oxidation through mask, c) mask removal to reveal waveguide

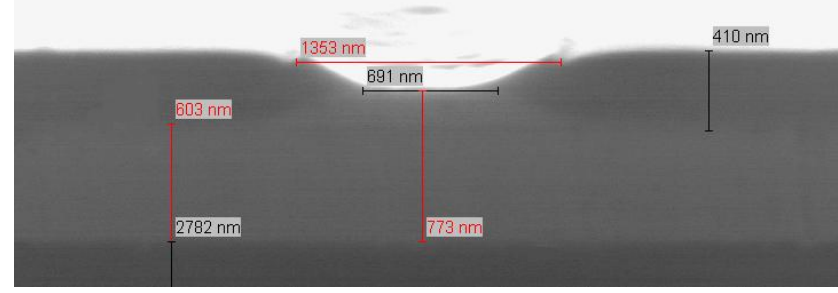

Fig: 2 SEM of the measured waveguides 


\section{MEASUREMENT METHOD}

The waveguides have been characterized in a standard free space optical measurement system. Prior to measurement, in order to couple the light efficiently into the waveguides, the end-facets were polished by lapping with abrasive materials with sequentially decreasing sizes, until optically smooth facets were achieved. An optical beam from a Photonetics TUNICS-PRI Benchtop Tunable Laser was coupled to the waveguides via a polarisation maintaining fibre and a broadband polarising cube beam splitter, in order to remove any residual TM polarisation component from the beam. Subsequently a half wave plate placed after the cube allows for the linear polarisation state of the input beam to be converted from TE to TM. The test chip was placed between two objective lenses used to focus the light into and collimate the light out of the waveguide. Finally, a linear polariser was used to filter the output light in order to characterise the power level for a given polarisation. The output power was then monitored through an Agilent 8164A Interface and $81625 \mathrm{~A}$ detector.

On the test chip, the facets were not anti-reflection coated such that the Fabry-Perot loss measurement technique could be utilized [3-5]. Therefore the light propagates along the waveguide and is reflected at either facets by an amount determined by the refractive index of the waveguide material (silicon) and the external material (air). Wavelength scans can then be used to determine the waveguide loss. Typical wavelength scans are shown in figure 3.

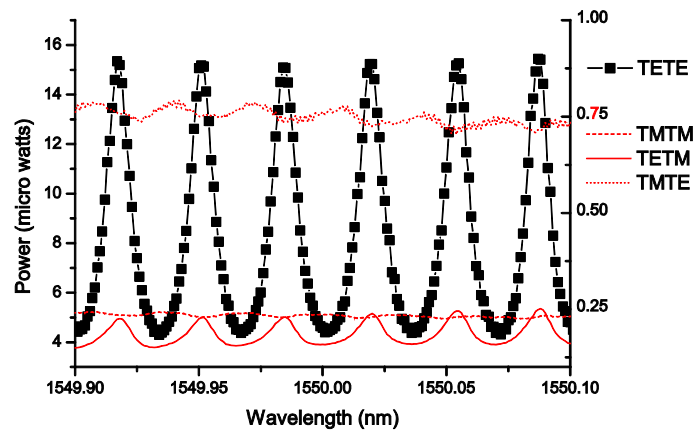

(a)

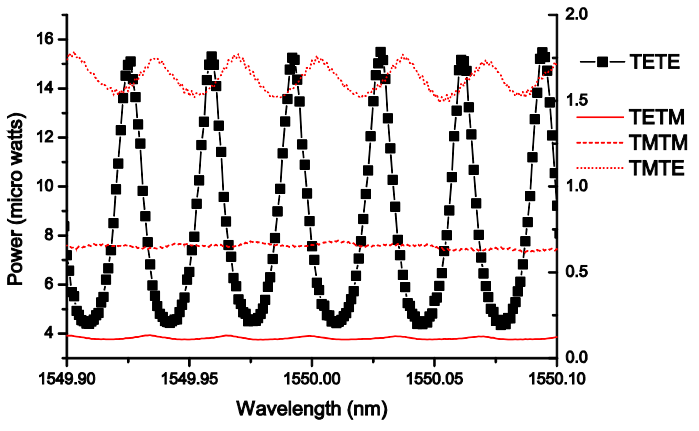

(b)

Fig: 3 Fabry Perrot wavelength scans for 2 waveguides with different mask widths a) 1.1 microns, b) 1.5 microns.

The optical loss has been determined for both TE and TM polarisation input states, and for each case, the output power was separated into both states in order to monitor any polarisation dependent loss. Fig. 4 shows the impact of the FabryPerrot cavity on the output signal for waveguide widths $1.1,1.5,1.7 \mu \mathrm{m}$ respectively, where the width is measured at the base of the waveguide side-walls. Each curve is labelled such that the input polarisation is followed by the measured output polarisation. The four curves are displayed on two different scales with TE-TE on the left, while for the rest the power scale is on the right. The loss measured for TE-TE polarisation, for a length of $9.5 \mathrm{~mm}$ was $0.32 \mathrm{~dB} / \mathrm{cm}, 0.21$ $\mathrm{dB} / \mathrm{cm}$, respectively for waveguide widths of 1.1 , and $1.5 \mu \mathrm{m}$ at a wavelength of $1550 \mathrm{~nm}$.

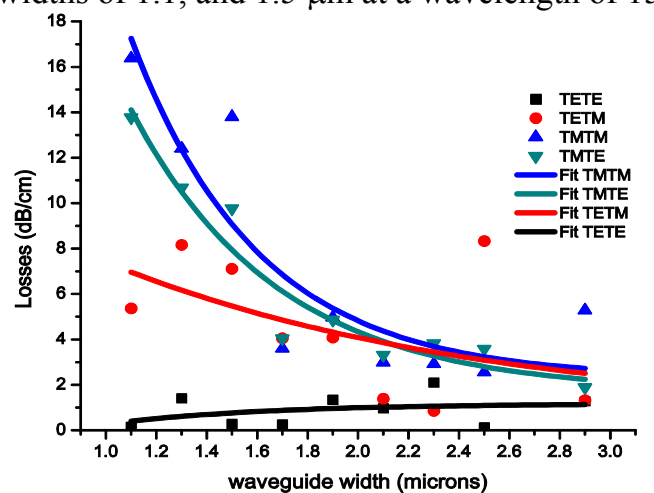

Fig: 4 Polarisation dependent loss with waveguide width. 
When the waveguide width is decreased the evolution of the interaction of the mode with the side walls is different for TE and TM polarisation. This has a direct impact on the losses, moreover LOCOS waveguides are formed of very pronounced sidewall angles, hence it was also possible to see polarisation conversion effects (to be discussed elsewhere). It is clear that pure TE transmission is almost unaffected by the variation of the width of the waveguide where the loss is in the 0.2 to $2 \mathrm{~dB} / \mathrm{cm}$ range. To the contrary, pure TM transmission is affected and varies from 3 $\mathrm{dB} / \mathrm{cm}$ to more than $15 \mathrm{~dB} / \mathrm{cm}$ for the lowest waveguide width measured .

\section{CONCLUSIONS}

This paper discusses the formation of optical waveguides by the LOCOS process. This is a well established process in microelectronics, and lead to the fabrication of rib waveguides with very smooth waveguide walls. However, the waveguide walls are formed with sidewalls at an angle of $22^{\circ}$ to the substrate which leads to significant variation in polarization performance in terms of loss and also purity. Nevertheless this is a very low cost process and results in sub micron waveguides with very low losses, as low as fractions of a $\mathrm{dB} / \mathrm{cm}$. Consequently these waveguides would be suitable for low loss, moderate volume applications. Furthermore, the fact that the waveguides can be so small means that they will have significant evanescent fields at the surface and thus would be ideal for low cost sensor applications.

\section{REFERENCES}

[1] L. K. Rowe, M. Elsey, N. G. Tarr, A. P. Knights, and E. Post, "CMOS-compatible optical rib waveguides defined by local oxidation of silicon," Electronics Letters, vol. 43, pp. 392-393, 2007.

[2] C. Kuang Yi, J. L. Moll, and J. Manoliu, "A bird's beak free local oxidation technology feasible for VLSI circuits fabrication," Electron Devices, IEEE Transactions on, vol. 29, pp. 536-540, 1982.

[3] G. T. Reed and A. P. Knights, Silicon Photonics: An Introduction: John Wiley \& Sons, Inc., 2004.

[4] A. Sakai, G. Hara, and T. Baba, "Propagation characteristics of ultrahigh-\&Delta; optical waveguide on silicon-on-insulator substrate," Japanese Journal of Applied Physics, Part 2: Letters, vol. 40, pp. 383-385, 2001.

[5] D. Duchesne, R. Morandotti, P. Cheben, B. Lamontagne, D. Xu, and S. Janz, "Waveguide birefringence in asymmetric silicon-on-insulator nanowires," Montreal, Que., 